\title{
LUMINOSITY FUNCTION AND SPATIAL DISTRIBUTION OF NEARBY GALAXIES
}

\author{
K.-H. SCHMIDT ${ }^{1}$ and T. BOLLER ${ }^{2}$ \\ ${ }^{1}$ Astrophysikalisches Institut, An der Sternwarte 16, Potsdam, Germany \\ ${ }^{2}$ Max-Planck-Institut für Extraterrestrische Physik, Karl-Schwarzschild-Strasse 2, \\ Garching, Germany
}

\section{Introduction}

The investigation of environmental effects is a topic of high priority in current extragalactic research. In this connection, the study of neighbouring galaxies will result in characteristic properties of a low-density sample of extragalactic systems which may be used for comparison with other samples, with clusters of galaxies, for instance. The advantage of nearby galaxies is that they allow us to obtain an almost complete sample down to low-luminous objects because of their small distances.

\section{The Sample and its Completeness}

All galaxies with measured redshifts smaller than $500 \mathrm{~km} / \mathrm{s}$ or at least with probable membership in one of the nearby groups have been compiled (Schmidt \& Boller 1992a). The revised catalogue of these nearby objects (Schmidt et al. 1993) contains 276 galaxies; of these, including the Milky Way System, 206 galaxies are within a sphere of $r=7.154 \mathrm{Mpc}$ assuming the distance to the Virgo cluster of $21.7 \mathrm{Mpc}$. 192 galaxies of the sample are brighter than $\mathbf{M}(\mathrm{B})=-10$.

The incompleteness of this sample is mainly caused by two reasons: 1) the failure of spectroscopic data of galaxies which have not been recognized as close by up to now, and 2) unknown galaxies hidden by the interstellar dust near the galactic plane.

The completeness is evaluated by assuming a homogeneous distribution of the galaxies in a flat disk around the supergalactic plane suggested by the apparent distribution of the objects and by estimating the mean distances of the known members of the sample. This consideration yields about 318 expected galaxies brighter than $M(B)=-10$, that means about 40 per cent of the objects within $r=7 \mathrm{Mpc}$ are unknown, most of them fainter than $M(B)=-13$. However, there may be an overestimate because the observed faint galaxies, which determine the statistics, belong mainly to the Local Group and the M 81 group, sites of enhanced density. Therefore, 30 to 35 per cent of unknown nearby galaxies is a more realistic number.

Among the nearby galaxies brighter than $M(B)=-18$, presumably only 2 have been not recognized so far. Thus, the mean density of galaxies brighter than $M(B)=-18$ within $7 \mathrm{Mpc}$ is $0.023 \mathrm{Mpc}^{-3}$. Compared with this the density of bright galaxies in the inner part of the Virgo 
cluster is about $4.2 \mathrm{Mpc}^{-3}$.

\section{Luminosity Function of Nearby Galaxies}

The distribution of absolute magnitudes of the galaxies of the $7 \mathrm{Mpc}$ sample is shown in Fig. 1 (solid histogram). Correcting this distribution for incompleteness, the dashed histogram is obtained which may be representative of the luminosity function of nearby galaxies neglecting the part which is hidden by the interstellar dust in the zone of avoidance. The observed luminosity function is best fitted by a Schechter (1976) function with parameters $\mathbf{M}^{*}=-21.5$ and $\alpha=-1.15$. These parameters are insignificantly different from previous results resting only on the bright part of the distribution (Schmidt \& Boller 1992b) as well as from those obtained by Tully (1988), but are in remarkable agreement with the result of van den Bergh (1992) for the Local Group.

For comparison Sandage et al. (1985) obtained $M^{*}=-21.1 \ldots-21.3$ and $\alpha=-1.25 \ldots-1.30$ for the Virgo cluster. Obviously there are no large differences between the global luminosity functions of samples exhibiting considerably different densities.

\section{Frequency of Different Morphological Type}

Plot of the absolute magnitude versus morphological type demonstrates the large frequency of spiral galaxies and irregular systems in the extragalactic neighbourhood of the Milky Way System. Comparing the morphological population of the nearby sample with that of the Virgo cluster the almost equal frequency of spiral and elliptical systems among the bright galaxies and the overwhelming frequency of the dwarf ellipticals in the Virgo cluster is striking.

Besides this clear difference between the two samples there is another noticeable feature. According to Sandage et al. (1985) the luminosity function of the irregular galaxies in the Virgo cluster fits a Schechter function with $\alpha=-0.25$ very well, whereas the same quantity for the

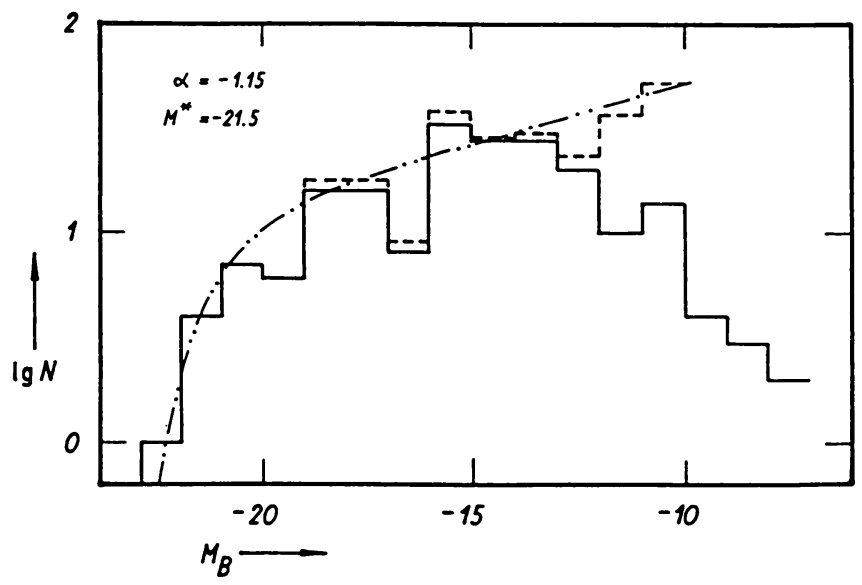

Figure 1. Luminosity function of the $7 \mathrm{Mpc}$ sample. 
luminosity function of the nearby irregulars is somewhere between -0.5 and -1.0 . Additionally the absolute magnitude of the maximum of the luminosity function of the nearby irregulars is fainter than for that of the Virgo irregulars by about 2 mag. It would appear that these facts are due to different environmental effects in both samples presumably caused by their different densities.

\section{Properties of Nearby Groups}

Looking at the apparent distribution of the nearby galaxies, 1) the concentration to the supergalactic equator and 2) the clumpiness of the distribution are striking phenomena. By the first observational fact de Vaucouleurs (1953) was caused to introduce the supergalactic coordinates, whereas the observed patchy distribution leads to the detection of groups of galaxies. Following the definition of nearby groups by Kraan-Korteweg \& Tammann (1979) half a dozen groups of galaxies are within the $7 \mathrm{Mpc}$ sample comprising roughly 80 per cent of the total known population of nearby galaxies.

From the morphological aspect we may discern at least two types of groups. The representative of the first class consists of a central concentration with diameter of about $0.5 \mathrm{Mpc}$ which is surrounded by a loose cloud of normally faint galaxies. Usually the most luminous member in a group is the head of the central concentration. A typical case of this class is the M 81 group.

The second type of groups is characterized by two concentrations similar to those of the first class. These two conglomerates which are separated by about $1 \mathrm{Mpc}$ are enveloped by a common cloud of faint galaxies. Representatives of this type are the Local Group with the concentrations around M 31 and the Milky Way System, and the Centaurus group with the concentrations around NGC 5128 and M83.

The richness of the groups may be expressed by the number of objects brighter than $M(B)=-15$. This number is between 6 and 15 for the nearby groups.

An interesting result is the correlation between the mean slope of the logarithm of the cumulative luminosity function of a group in the range $-20<M(B)<-14$ and the earliest morphological type among the brighter members of a group. Obviously the field galaxies do not obey this correlation. We may expect that the observed relation is greatly based on a special property, which is due to the morphological type of a galaxy and may be a hint on environmental effects or formation processes. Such a quantity is the specific angular momentum which increases from S0 to Sm Hubble types (Mineva 1988). According to Busarello et al. (1993) the angular momentum of elliptical galaxies is a factor 6 lower than for disk galaxies of the same mass. An explanation of the observed relation between the slope of the cumulative luminosity function and the earliest morphological type of a member galaxy may be given by a scenario in which a pregalactic cloud exhibiting a small specific angular momentum decays mainly into more massive objects than a cloud with the mass but larger angular momentum for which the accumulation to larger entities is hampered. 


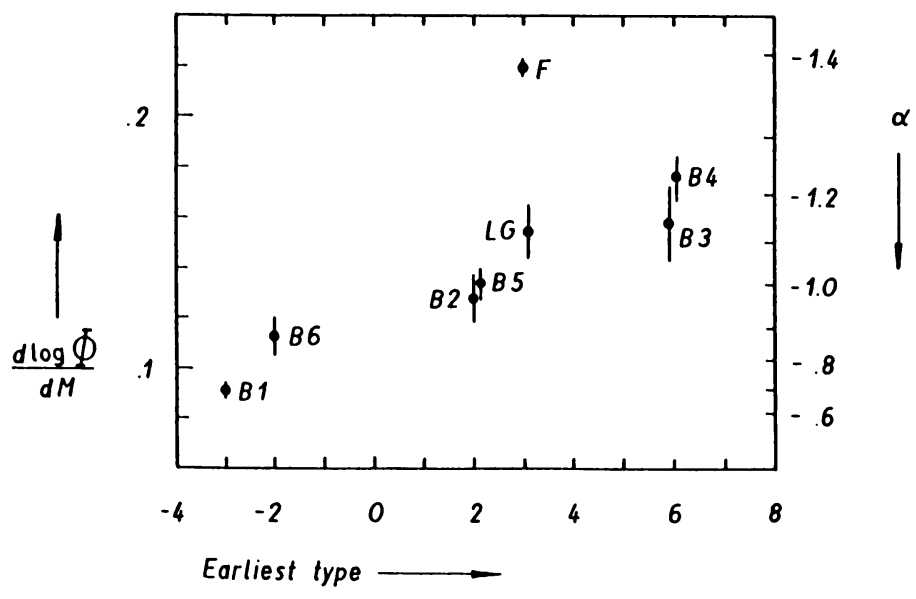

Figure 2. Relation between the slope of the luminosity functions of several groups of nearby galaxies in dependence on the earliest morphological type among the bright members of the group considered. The designations are from Kraan-Korteweg \& Tammann (1979). 'LG' stands for 'Local Group', 'F' for field galaxies.

\section{Density Distribution Perpendicular to the Supergalactic Plane}

The apparent distribution of the nearby galaxies shows a concentration to the supergalactic plane. The histogram of the distances $\mathrm{Z}$ of the galaxies from the supergalactic plane confirms this impression (Fig. 3). This $\mathrm{Z}$ distribution is fitted well by a Gaussian distribution supplemented by a small constant term. Thus, the half-density thickness of the layer in which the nearby galaxies considered here are concentrated is smaller than $1 \mathrm{Mpc}$. Clearly the main population of

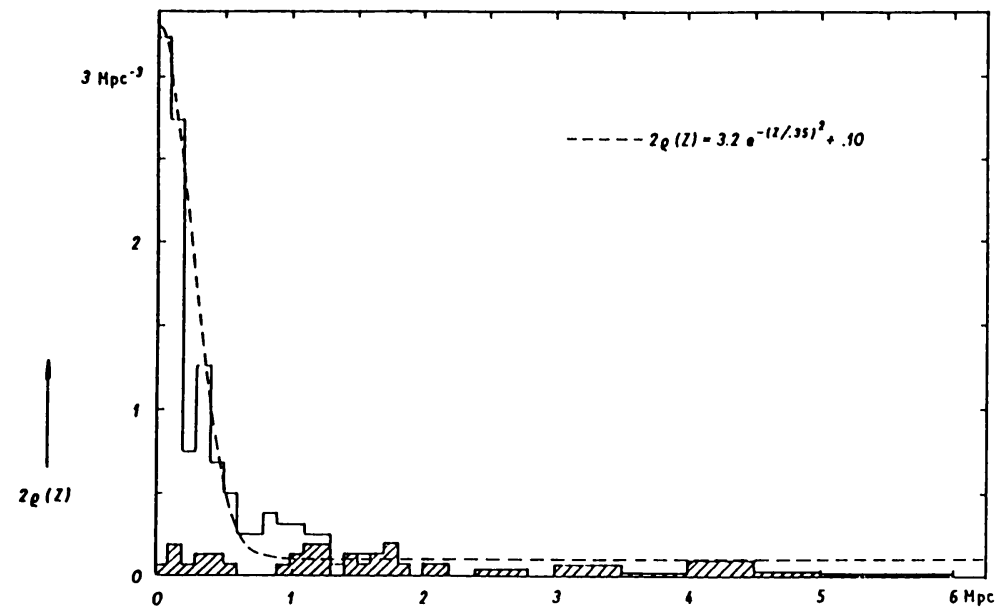

Figure 3. Histogram of the distances perpendicular to the supergalactic plane of the galaxies of the $7 \mathrm{Mpc}$ sample. The field galaxies are marked by the hatched area. 
this disk is due to members of the groups: there is no group member within $r=7 \mathrm{Mpc}$ at a larger distance perpendicular to the supergalactic plane than $1.6 \mathrm{Mpc}$.

Obviously the disk in the supergalactic plane separates two voids from each other which are below and above the plane. But these voids are distinctly not empty as suggested by the additive term in the fitting curve to the observed distribution. This term may be interpreted by the contribution of the field galaxies (hatched in Fig. 3 ) which is nearly constant if one considers the incompleteness at larger distances.

\section{References}

Busarello, G., Longo, G. and Feoli, A., 1993. Astron. Astrophys., in press.

de Vaucouleurs, G., 1953. Astron. J., 58, 30.

Kraan-Korteweg, R.C. and Tammann, G.A., 1979. Astron. Nachr., 300, 181.

Mineva, V.A., 1988. Astron. Zh., 65, 702.

Sandage, A., Binggeli, B. and Tammann, G.A., 1985. Astron. J., 90, 1759.

Schechter, P.L., 1976. Astrophys. J., 203, 297.

Schmidt, K.-H. and Boller, T., 1992a. Astron. Nachr., 313189.

Schmidt, K.-H. and Boller, T., 1992b. Astron. Nachr., 313, 329.

Schmidt, K.-H., Priebe, A. and Boller, T., 1993. Astron. Nachr., in press.

Tully, R.B., 1988. Astron. J., 96, 73.

van den Bergh, S., 1992. Astron. Astrophys., 264, 75. 